\title{
Phytoprotection
}

\section{Liste des arbitres 1988-1989}

\section{List of reviewers 1988-1989}

Volume 71, numéro 2, 1990

URI : https://id.erudit.org/iderudit/705990ar

DOI : https://doi.org/10.7202/705990ar

Aller au sommaire du numéro

\section{Éditeur(s)}

Société de protection des plantes du Québec (SPPQ)l

\section{ISSN}

0031-9511 (imprimé)

1710-1603 (numérique)

Découvrir la revue

Citer ce document

(1990). Liste des arbitres 1988-1989. Phytoprotection, 71(2), 105-106.

https://doi.org/10.7202/705990ar d'utilisation que vous pouvez consulter en ligne.

https://apropos.erudit.org/fr/usagers/politique-dutilisation/ 
Alforo, R.

Asselin, A.

Baldwin, R.E.

Bélair, G.

Benhamou, $N$.

Bloomberg, W.J.

Boiteau, G.

Chalifour, F.P.

Charest, P.M.

Chiykowski, L.N.

Cloutier, D.

Dalpé, Y.

Danks, H.V.

Dion, $P$.

Dumas, M.T.

Giroux, $M$.

Granger, R.

Gray, A.B.

Haber, S.M.

Hall, R.

Hamilton, R.I.

Hildebrand, P.D.

Hogue, R.

Hussey, R.S.

Jaques, R.P.

Kerber, E.R.

Kimpinski, J.

Kushalappa, A.C.

Lachance, R.A.

Lalonde, $M$.

Leach, S.S.

Légère, $A$.

Lemieux, C.

Leroux, G.

Mather, D.E.

Michaud, S.G.

Morrall, R.A.A.

Morris, O.N.

Neumann, $P$.

Olthof, T.H.A.

Otrysko, B.E.

Ouellette, G.B.

Parrella, M.P.

Parent, J.-G.

Pelletier, J.-R.

Peterson, J.F.

Prévost, Y.

Reeleder, R.D.

Ring, R.A.

Rioux, R.

Roy, $M$.

Sackston, W.E.

Sauriol, P.

Smith, R.F.

Sparace, S.A.

Sutton, J.C.

Sweeney, J.

Tartier, L.

Therrien, P.

Thomas, A.G.

Turgeon, J.

Tyson, $\mathrm{H}$.
Service canadien des forêts, Vancouver (British Columbia)

Université Laval, Québec (Québec)

Virginia State University, Painter (Virginia)

Agriculture Canada, Saint-Jean-sur-Richelieu (Québec)

Université Laval, Québec (Québec)

Service canadien des forêts, Vancouver (British Columbia)

Agriculture Canada, Fredericton (New Brunswick)

Université Laval, Québec (Québec)

Université Laval, Québec (Québec)

Agriculture Canada, Ottawa (Ontario)

Agriculture Canada, L'Assomption (Québec)

Agriculture Canada, Ottawa (Ontario)

Musée d'histoire naturelle, Ottawa (Ontario)

Université Laval, Québec (Québec)

Service canadien des forêts, Sault-Sainte-Marie (Ontario)

MAPAQ, Québec (Québec)

Agriculture Canada, Saint-Jean-sur-Richelieu (Québec)

Nova Scotia Agricultural College, Truro (Nova Scotia)

Agriculture Canada, Winnipeg (Manitoba)

University of Guelph, Guelph (Ontario)

Agriculture Canada, Vancouver (British Columbia)

Agriculture Canada, Kentville (Nova Scotia)

MAPAQ, Québec (Québec)

University of Guelph, Guelph (Ontario)

Agriculture Canada, Harrow (Ontario)

Agriculture Canada, Winnipeg (Manitoba)

Agriculture Canada, Charlottetown (Prince Edward Island)

Macdonald College, Sainte-Anne-de-Bellevue (Québec)

Université Laval, Québec (Québec)

Université Laval, Québec (Québec)

University of Maine, Orono (Maine)

Agriculture Canada, Sainte-Foy (Québec)

Agriculture Canada, Sainte-Foy (Québec)

Université Laval, Québec (Québec)

Macdonald College, Sainte-Anne-de-Bellevue (Québec)

New Brunswick Dept. of Agriculture, Fredericton (New Brunswick)

University of Saskatchewan, Saskatoon (Saskatchewan)

Agriculture Canada, Winnipeg (Manitoba)

Université de Montréal, Montréal (Québec)

Agriculture Canada, Vineland (Ontario)

Université Laval, Québec (Québec)

Service canadien des forêts, Sainte-Foy (Québec)

University of California, Riverside (California)

Université Laval, Québec (Québec)

Agriculture Canada, Saint-Jean-sur-Richelieu (Québec)

Macdonald College, Sainte-Anne-de-Bellevue (Québec)

Lakehead University, Thunderbay (Ontario)

Macdonald College, Sainte-Anne-de-Bellevue (Québec)

University of Victoria, Victoria (British Columbia)

Agriculture Canada, La Pocatière (Québec)

MAPAQ, Saint-Hyacinthe (Québec)

Macdonald College, Sainte-Anne-de-Bellevue (Québec)

M.A.P.A.Q., Saint-Rémi (Québec)

Agriculture Canada, Kentville (Nova Scotia)

Macdonald College, Sainte-Anne-de-Bellevue (Québec)

University of Guelph, Guelph (Ontario)

Pacific Forestry Center, Victoria (British Columbia)

MAPAQ, Saint-Hyacinthe (Québec)

Ministère de l'Énergie et des Ressources, Québec (Québec)

Agriculture Canada, Régina (Saskatchewan)

Service canadien des forêts, Sault-Sainte-Marie (Ontario)

McGill University, Montréal (Québec) 
Vincent, C.

Vrain, T.C.

Weaver, S.E.

Whitfield G.H.

Whitney, R.D.

Zoebish, T.G.
Agriculture Canada, Saint-Jean-sur-Richelieu (Québec)

Agriculture Canada, Vancouver (British Columbia)

Agriculture Canada, Harrow (Ontario)

Agriculture Canada, Harrow (Ontario)

Service canadien des forêts, Sault-Sainte-Marie (Ontario)

University of Florida, Bradenton (Florida)

Centre agricole Bienvenue (Rougemont) Inc., Rougemont (Québec)

Ciba Geigy Canada Ltd., Mississauga (Ontario)

Standish Bros. Ltd., Cookshire (Québec)

W.H. Perron E Cie Ltée, Laval (Québec)

United Agri Products (UAP), Ville Saint-Laurent (Québec)

Nutrite Inc., Brossard (Québec)

Coopérative fédérée de Québec, Montréal (Québec) 